\title{
Development of a Lidar System Based on an Infrared RangeFinder Sensor and SlipRing Mechanism
}

\author{
Gokhan Bayar**, Alparslan Uludag* \\ *Department of Mechanical Engineering, Faculty of Engineering, Bulent Ecevit University, 67100 Zonguldak, Turkey. \\ (bayar@beun.edu.tr, alpadras@gmail.com) \\ ${ }^{\sharp}$ Corresponding Author; Gokhan Bayar, Department of Mechanical Engineering, Faculty of Engineering, Bulent Ecevit \\ University, 67100 Zonguldak, Turkey, Tel: +90 3722574010/1224, Fax: +90 3722574023, email: bayar@ beun.edu.tr
}

Received: 06.06.2016 Accepted: 03.08.2016

\begin{abstract}
Lidar systems are one of the most important sensor infrastructures in autonomous vehicles and mobile robots. They are used for achieving indoor and outdoor mapping purposes. In the scope of this study, a new perspective to develop a lidar system is proposed. The system developed is constructed based on a low-cost infrared rangefinder sensor, low-cost slipring mechanism designed and manufactured, dc motor and microprocessor. The rangefinder sensor is mounted to a head-structure actuated by a dc motor that continuously rotates with a desired rotational velocity. The data coming from the rangefinder sensor flows through the microprocessor via the slipring. The design concept of the slipring mechanism gives an advantage that the data of the infrared rangefinder sensor can be taken while the sensor continuously rotates. The required power for the sensor can also be supplied by the slipring during motion. The decoding process of the data coming from the rangefinder sensor and motor control task are accomplished using an ATmega based microprocessor. A user interface is also created to communicate with the system and evaluate the performance of the whole structure developed. After conducting many experiments, successful results are obtained. The design steps of the system proposed and the experimental results are presented in this paper.
\end{abstract}

Keywords Lidar, infrared sensor, slipring, laser scanner, range finder.

\section{Introduction}

Autonomous vehicles and mobile robots require recognizing their surroundings. They are generally programmed to track a predefined trajectory / path in order to perform an autonomous task. While they effort to track a reference trajectory, a lidar system provides them the surrounding information (in order words lidar system behaves like their eyes). By this way, they can be able to recognize the obstacles, people, other vehicles, etc. The use of information coming from a lidar system can provide the opportunities to the autonomous systems to take some pre-actions so that any unexpected events could be prevented. In addition to recognizing the working environment of the autonomous vehicles, lidar systems are also used as a feedback sensor for the control systems. Desired trajectory tracking tasks including position, orientation and velocity control routines can be supported by the feedback information acquired from a lidar system.
In the autonomous vehicle applications, path planning is one of the most critical issues that should be carefully designed. To achieve a successful path tracking control, reference path should be constructed by taking into account the working environment structure, capabilities and capacities of the vehicle and sensors. Lidar systems are one of the solutions for developing real-time maps of the working area and planning the desired paths. They can be adapted into the path planning algorithms as a powerful feedback option. The feedback information can also be used for the localization issues. An autonomous vehicle application, in which the location of the vehicle during the motion should be known, requires real-time position and orientation information. This can also be achieved by the use of a lidar system. An autonomous orchard application may be a good example for such a task (as presented in Fig. 1). In this figure, an orchard environment including trees and rows of trees and a four-wheeled vehicle is shown. In this scenario, the vehicle should drive itself autonomously inside the orchard. A lidar is mounted at the 
front-mid-center of the vehicle for recognizing the surroundings. Its responsibilities are to provide the required data which is used for both detecting trees and estimating rows of trees. The lidar data is also necessary for localizing the vehicle inside the orchard. Suppose that point $\left(\mathrm{X}_{0}, \mathrm{Y}_{0}\right)$ is the starting position. The scanning radius of the lidar is shown by $\mathrm{R}$. In order to create a safe drive, let's say this is an objective in this scenario, the vehicle should move on the center line that is exactly the middle of two neighboring rows. When there is a positioning error, indicated by $\mathrm{y}_{\mathrm{e}}$ in Fig. 1, the lidar system should warn the control system to take the required actions for steering the vehicle through the desired path. This action is also depicted in Fig. 1.

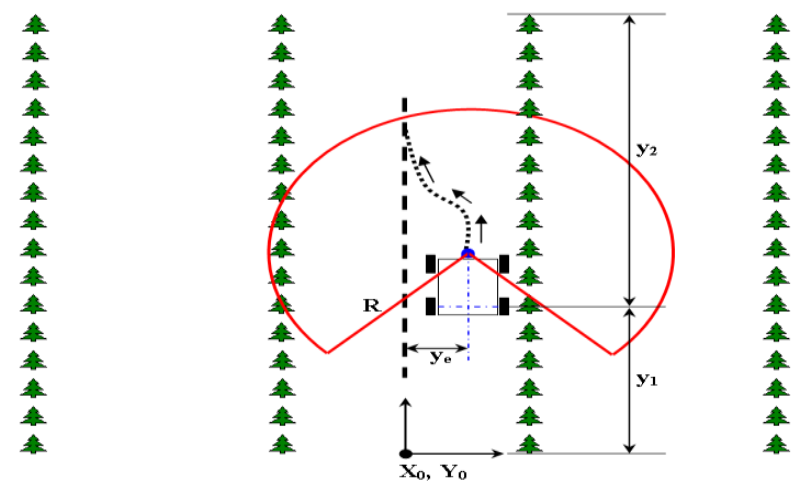

Fig. 1. The use of lidar in an autonomous orchard application

The importance of the use of lidar systems in autonomous applications is shown by a couple of examples presented above. Development of new lidar systems, approaches and models is still being continued. Parallel to the enhancements in the lidar research area, a new methodology for developing a lidar system is focused in this study. The new methodology involves the adaptation of a low-cost infrared rangefinder and a low-cost and easyto-use slipring mechanism. The rangefinder sensor, which continuously rotates by the help of a head structure designed, is used to scan the $360^{\circ}$ surrounding of the lidar system developed. This rotating mechanism enables that the working environment of the sensor can be sensed. Furthermore, when the sensor is surrounded by an object, the $2 \mathrm{D}$ shape of the object can be detected. The rotational motion is provided using a dc motor. The slipring mechanism is plugged to the system for achieving data flow from the rangefinder sensor to the microprocessor. ATmega based microprocessor is also used to achieve computation and communication purposes.

This paper is organized as follows: the next section presents the literature studies reviewed. Section 3 presents the lidar systems and their working principles. Section 4 introduces the lidar system proposed in this study. Prior to the concluding remarks, experimental results are given in Section 5.

\section{Literature Studies}

The studies related to lidar systems can be reviewed into two main topics. The first topic is about proposing new approaches to develop lidar systems. The other topic focuses on implementing the lidar systems into the robotic applications.

In [1], a lidar application for agricultural tasks was proposed. The data coming from a lidar was the only sensor source that provides feedback information to the mathematical model developed. In the scope of this study, a lidar canopy height model was developed using a semiautomated pit filling algorithm. In [2], the accuracy enhancement of large scale canopy heights was focused. A new approach based on lidar output was. The system introduced was tested by the help of different scenarios. In [3], lidar system was used in city modeling and building recognition tasks. The use of methodology introduced for building footprints was presented. Classification of the roof structures of the buildings was also evaluated using algorithm created based on the lidar sensor data. In [4], lidar sensor was used for a planetary application. A system based on the lidar sensor data was constructed for achieving autonomous safe landing on planetary bodies. In [5], a lidar system was developed for forestry applications. The methodology was constructed based on laser induced fluorescence imaging technique. The system developed was tested to observe the performance of the proposed structure. Working characteristics of the system was also produced. In [6], a lidar was used to estimate individual tree heights in forestry applications. The data coming form lidar was accompanied with the aerial photography. In [7], a new approach for detecting stone monuments was proposed. The method was constructed based on data coming from a lidar. In [8], a lidar system was developed for observing the atmospheric events and the particle density inside in it. In [9], a crop monitoring system was proposed. The system was constructed based on a lidar and 3D stereoscopic vision system. The system developed was tested for autonomous agricultural applications. In [10], a lidar was placed on a mobile platform and the data was logged. The developed methodology used the data logged for extracting highway light poles and towers. In [11], an automatic classification of urban pavements system based on lidar was built. In [12], a lidar based intelligent system was developed for recognizing the ancient city walls. The modeling strategy was also was used for doing digital documentation. In [13], a road detection system was constructed using lidar point clouds. The system was modeled in a way that could have the capability for adapting itself according to the data intensity. In [14], a lidar based system was built for detecting open water surfaces in an Arctic delta. The system was modeled by following the principles of decision tree classification technique.

As seen in the literature studies, lidar systems are commonly used in different applications ranging from defense to industrial robotics, agriculture to automation, unmanned ground vehicles to submarines, civil engineering to architecture. In the market, there are already some lidar systems $[15,16,17,18]$ that are ready-to-use for the researchers and engineers. However, they may not be affordable for small-scale projects, small-scale research companies, student works, startup formations, etc. Due to 
their high-prices, many lidar development research studies have been continuing. Their common goal is to build lowprice and easy-to-implement lidar systems. In parallel with the recent developments in the lidar research, this study aims to make a contribution to the continued investigations about developing lidar systems and to create a new perspective for this field.

\section{Lidar Systems}

Lidar systems are commonly used in different research studies and industrial applications. They are adapted into the real-time running unmanned aerial and autonomous ground vehicles. Path planning, mapping and control issues are supported by the data coming from lidar systems. They are also used for terrestrial scanning. Topography map creation, mining reconstruction, architecture, archaeology, building researches and city reconstruction require environmental data supplied by a lidar. Lidar systems are also intensively used for observation of agricultural and forestry areas, urban sites, industrial and power plants. Real-time mapping of such regions can be created using the information provided by the lidar systems. The moving platforms like industrial forklifts, public transportation vehicles, trains, boats, offroad and unmanned vehicles are also suited with the lidar systems so as to ensure safe drive. In addition to providing fast drive and safe product handling, such systems are also utilized for increasing the reliability and performance in manufacturing systems. They are also implemented to the tools and systems manufactured by the defense industries. The other usage area of the lidar systems is the marine industry. Lidar systems can be plugged into any marine platforms to increase safe drive capabilities and working performance. Creating autonomy in marine products needs to be used lidar system as well.

A 2D lidar, laser scanning, system is depicted in Fig. 2. The system consists of a light source, transmitter, receiver and mirror. The light hitting the object and returning back is sensed by the receiver and the distance between the light source and the object is obtained. If an actuator is coupled to this system, a rotating lidar system would be built.
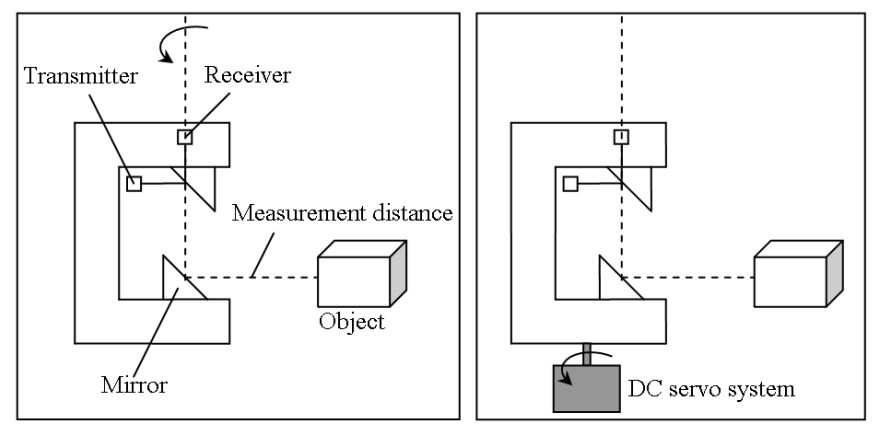

Fig. 2. Working principle of a lidar (laser scanning) system (left). Using an actuator to develop a rotating-lidar system (right)

\section{Lidar System Developed in This Study}

In this study, a design strategy to develop a lidar system is formed to meet the following criteria that the end product should be low-cost and accessible, easy-to-use and efficient. Following these design criteria, the lidar system consisting of three main subsections is taken into consideration. A low-cost infrared rangefinder sensor (Sharp GP2Y0A21YK) that can be easily found in the market is used. The Lidar system developed is able to detect the objects that are closer than $80 \mathrm{~cm}$. To trigger the sensor, $5 \mathrm{~V}$ DC voltage is required. It gives analog voltage as the output which should be decoded using a microprocessor. The block diagram of the system is shown in Fig. 3. In this figure, the reference input and outputs, data flows from sensors through the microprocessor and computer are shown. The control system constructed to control the dc actuator is also presented in this block diagram.

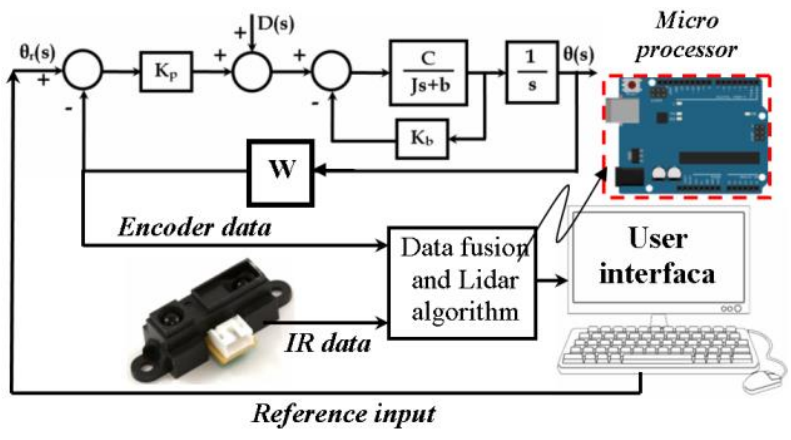

Fig. 3. Block diagram of the system developed

The infrared rangefinder sensor is mounted to a designed head-structure that continuously rotates (Fig. 4). The rotation is provided using a $6 \mathrm{~V}$ DC motor driven by a L298 H-bridge (motor motion control unit). The torque regulation is accomplished via a gear-head attached to the DC motor. An ATmega based microprocessor, called computing unit, is used for decoding, computing and communication purposes (Fig. 4). It needs 5V DC supply voltage and is connected through the computer via USB.

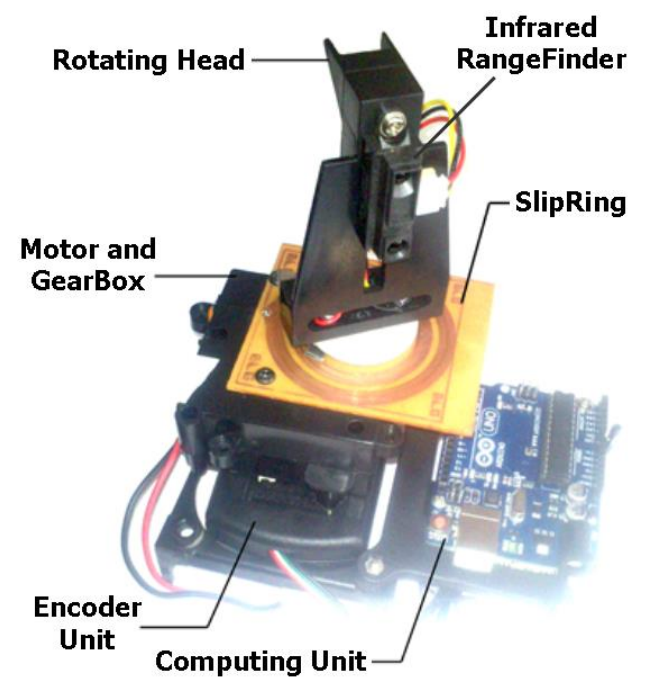

Fig. 4. General view of the lidar system developed in this study. Computing unit consists of an ATmega based microprocessor 
To get the rotation information, a simple encoder unit presented in Fig. 5 is also built. This unit consists of an encoder disc and Omron photomicrosensor [19]. The resolution of the encoder unit is 36 pulses per revolution. The counting of the pulses is also achieved using the ATmega based computing unit.

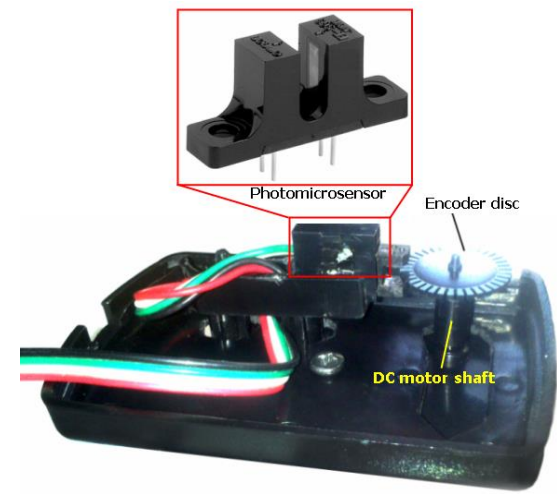

Fig. 5. Encoder unit including an encoder disc and photomicrosensor

In order to achieve data flow from the infrared rangefinder sensor, which continuously rotates during operation, to the computing unit, a new design perspective for slipring mechanism is considered. The top and side views of the developed system are shown in Fig. 6. The infrared rangefinder sensor needs three cable connections; one is for data flow and the other two is for power supply. The simple slipring mechanism, presented in Fig. 6, is constructed to make these connections. The data flow is achieved using the inner circle and the power is supplied via the middle and the outer circles (Fig. 6-left).
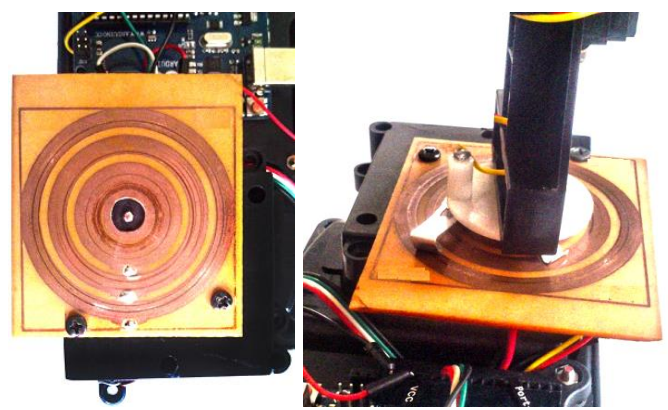

Fig. 6. Slipring mechanism developed. Top view (left) and side view (right) are shown.

\section{Experimental Studies}

In order to see the performance and accuracy of the lidar system proposed, the sensor structure introduced above is experimentally tested. It is placed at the center of a box which has the dimensions of $30 \mathrm{~cm} \times 21 \mathrm{~cm}$. The first experiment is performed for only 1 complete revolution. The resolution of the lidar system developed is 10 degrees therefore 36 distance measurements are obtained for one revolution. The results for this scenario are presented in Fig. 7 (top-left). To see the performance and accuracy enhancement while the number of turns is increased, experiments are performed from one to ten complete revolutions. The results obtained for "i" revolution(s), $(i=1,2, \ldots, 9)$, are shown in Fig. 7.

The experiment results including 10 full revolutions are given in Fig. 8. The results are presented in polar plots. The left polar plot shows all the measurements of the 10 turns whereas the right polar plot presents the average values of each measurement.

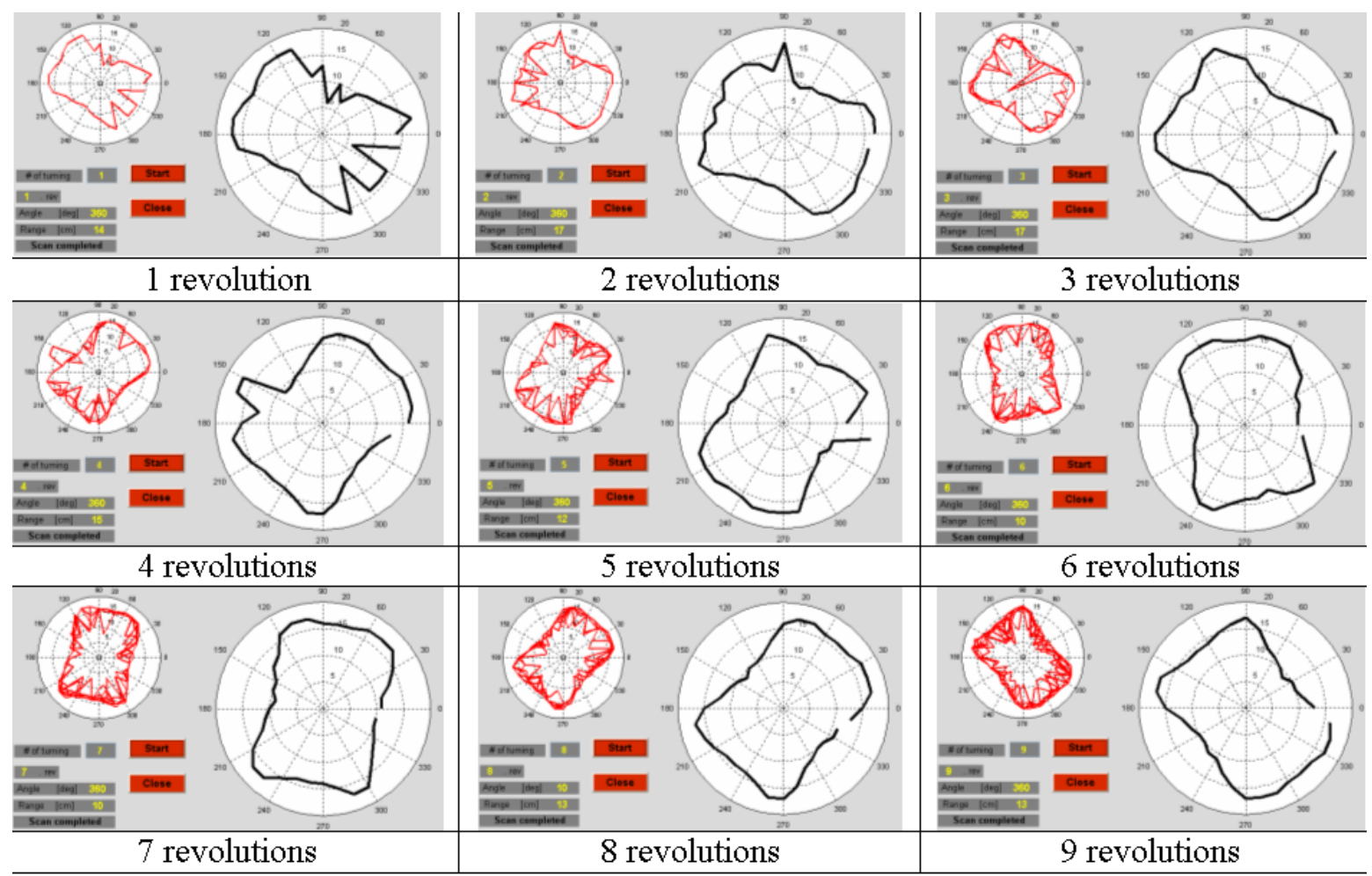

Fig. 7. Experiments performed using the lidar system. The results including 1 to 9 complete revolutions are presented 


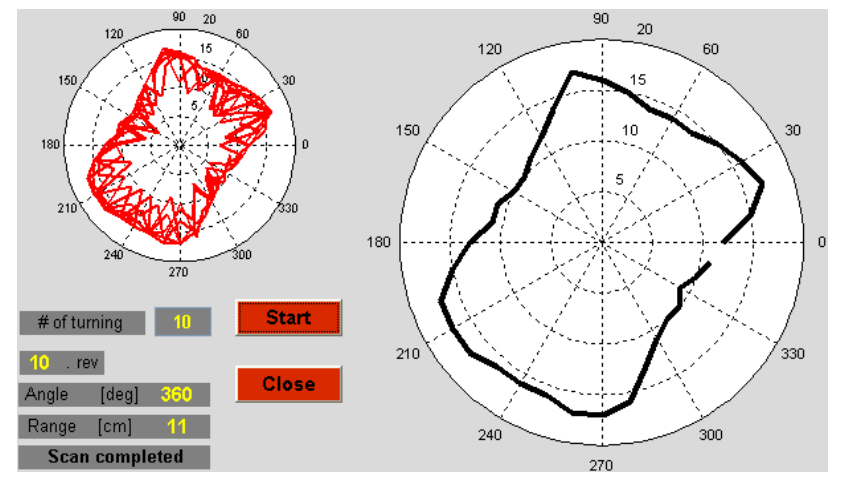

Fig. 8. Experiment results including 10 full revolutions. The left polar plot shows the measurement for all turns. The right polar plot indicates the average values of the measurements

The results given in Fig. 8 and 9 show that increasing the number of scans increases the accuracy and performance. The box's shape can be poorly estimated if only 1 revolution is performed (Fig. 9-a) whereas the shape obtained by the 10 complete revolutions is able to give almost the actual shape of the box (Fig. 9-b). These results emphasize that a lidar system developed using a low-cost infrared rangefinder sensor, a rotating headstructure and a simple slipring mechanism can be successfully used for scanning and recognizing purposes.

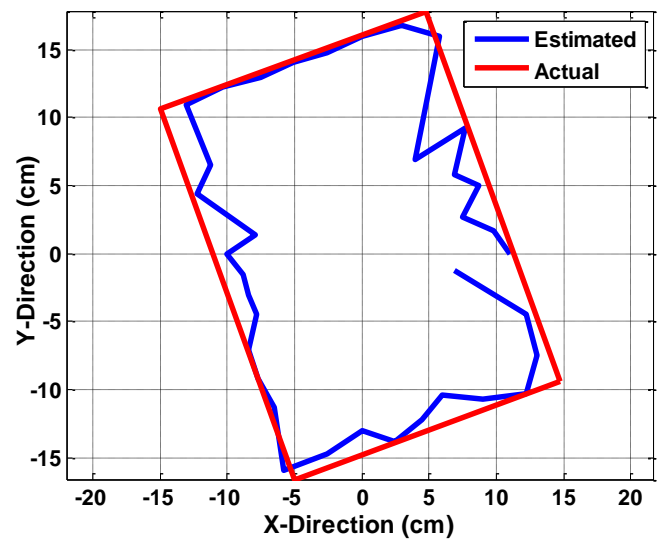

(a)

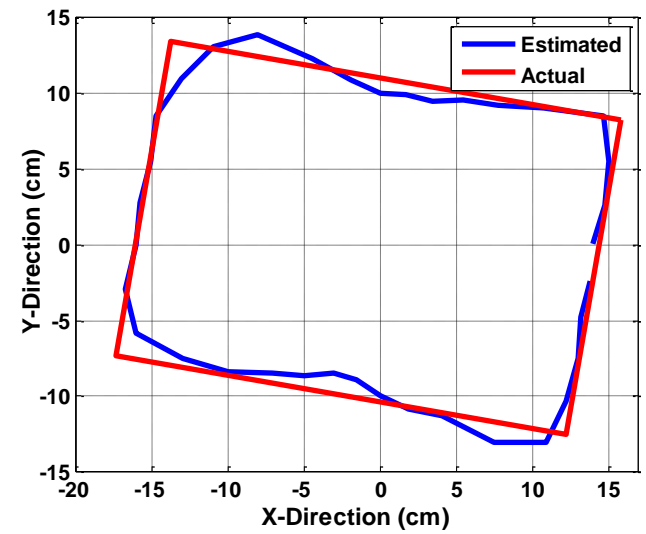

(b)

Fig. 9. The measurements of 1 revolution (a) and 10 revolutions (b). The red-colored-lines indicate the actual box's shape that has the dimensions of $30 \mathrm{~cm} \times 21 \mathrm{~cm}$. The

blue-colored-lines indicate the experimental results

\section{Analysis and Conclusion}

In indoor and outdoor mapping applications used for autonomous drives and automations, one of the important sensorial systems is the lidar. They are adapted into the control systems for providing feedback information. Lidar systems are able to scan the surrounding from 0 to 360 degrees in variety of distances. The distances may range from 0 to a few kilometers. In the market, there are already some lidar systems that are used for autonomous research and applications. However, these products may not be accessible by the small companies, small research groups and students because of their high-cost. In the scope of this study, it is objected to develop a low-cost, easy-to-use and easy-to-implement lidar system. The system proposed is built using a low-cost infrared rangefinder sensor and a slipring mechanism designed. The end product is able to rotate continuously and give distance data in every 10 degrees. The rotation is provided via a dc motor and the rotation information is obtained using an encoder. An ATmega based microprocessor is also used as the computational unit of the lidar system. In order to see the accuracy and the performance of the system proposed, a user interface is created in the computer side. Many experiments are performed and successful results are obtained. The results obtained in this study emphasize that the design strategy introduced provides a new perspective for developing lidar systems and can be used for the further researches.

\section{References}

[1] J. R. Ben-Arie, G. J.Hay, R. P. Powers, G. Castilla, B. St-Onge, Development of a pit filling algorithm for LiDAR canopy height models, Computers \& Geosciences, Vol. 35, pp. 1940-1949, 2009.

[2] M. Hollaus, W. Wagner, C. Eberhöfer, W. Karel, Accuracy of large-scale canopy heights derived from LiDAR data under operational constraints in a complex alpine environment, ISPRS Journal of Photogrammetry \& Remote Sensing, Vol. 60, pp. 323-338, 2006.

[3] C. Alexander, S. Smith-Voysey, C. Jarvis, Kevin Tansey, Integrating building footprints and LiDAR elevation data to classify roof structures and visualise buildings, Computers, Environment and Urban Systems, Vol. 33, pp. 285-292, 2009.

[4] F. Amzajerdian, D. Pierrottet, L. Petway, M. Vanek, Development of lidar sensor systems for autonomous safe landing on planetary bodies, International Conference on Space Optics, Rhodes Island, Greece, 2010

[5] Y. Saito, R. Saito, T. D. Kawahara, A. Nomura, S. Takeda, Development and performance characteristics of laser-induced fuorescence imaging lidar for forestry applications, Forest Ecology and Management, Vol. 128, pp. 129-137, 2000. 
[6] J. C. Suarez, C. Ontiveros, S. Smith, S. Snape, Use of airborne LiDAR andaerial photography in the estimation of individual tree heights in forestry, Computers \& Geosciences, Vol. 31, pp. 253-262, 2005.

[7] G. Cecchi, L. Pantani, V. Raimondi, L. Tomaselli, G. Lamenti, P. Tiano, R. Chiari, Fluorescence lidar technique for the remote sensing of stone monuments, Journal of Cultural Heritage, Vol. 1, pp. 29-36, 2000.

[8] J. Bregeon, M. Compin, S. Rivoire, M. Sanguillon, G. Vasileiadis, An elastic lidar system for the H.E.S.S. Experiment, Nuclear Instruments and Methods in Physics Research A, Vol. 819, pp. 60-66, 2016.

[9] M. Bietresato, G. Carabin, R. Vidoni, A. Gasparetto, F. Mazzetto, Evaluation of a LiDAR-based 3Dstereoscopic vision system for crop-monitoring applications, Computers and Electronics in Agriculture, Vol. 124, pp. 1-13, 2016.

[10] W. Y. Yan, S. Morsy, A. Shaker, M. Tulloch, Automatic extraction of highway light poles and towers from mobile LiDAR data, Optics \&LaserTechnology, Vol. 77, pp. 62-168, 2016.

[11] L. D. Vilariño, H. G. Jorge, M. Bueno, P. Arias, I. Puente, Automatic classification of urban pavements using mobile LiDAR data and roughness descriptors, Construction and Building Materials, Vol. 102, pp. 208-215, 2016.

[12] L. Cheng, Y. Wang, Y. Chen, M. Li, Using LiDAR for digital documentation of ancient city walls, Journal of Cultural Heritage, Vol. 17, pp. 188-193, 2016.

[13] Y. Li, B. Yong, H. Wu, R. An, H. Xu, Road detection from airborne LiDAR point clouds adaptivefor variability of intensity data, Optik, Vol. 126, 4292-4298, 2015.

[14] N. Crasto, C. Hopkinson, D. L. Forbes, L. Lesack, P. Marsh, I. Spooner, J. J. V. D. Sanden, A LiDARbased decision-tree classification of open water surfaces in an Arctic delta, Remote Sensing of Environment, Vol. 164, pp. 90-102, 2015.

[15] http://www.rieglusa.com/lidar-scanners-andsensors-systems.html, Last accessed, June 05, 2016.

[16] http://www.phoenix-aerial.com/products/lidarsystems, Last accessed, June 05, 2016.

[17] http://velodynelidar.com, Last accessed, June 05, 2016.

[18] http://www.teledyneoptech.com/index.php/products /airborne-survey/lidar-systems, Last accessed, June 05, 2016.

[19] https://www.omron.com/ecb/products/photo/\#photo micro, Last accessed, June 05, 2016. 